\title{
Multibody System Discrete Time Transfer Matrix Method for Nonlinear Shear Dynamic analysis of Immersed Tunnels
}

\author{
Zhongyuan Shen ${ }^{1, *}$, and Xue Bai ${ }^{1}$ \\ ${ }^{1}$ School of Civil and Transportation Engineering, Shanghai Urban Construction Vocational College, Shanghai, 201999, China
}

\begin{abstract}
Shear seismic response analysis is critical for seismic design of immersed tunnels. According to the structural characters of immersed tunnels and shear dynamic response of their joints, a multibody dynamic model consisting of multi-rigid body, shear hinge, and viscous damping hinge is proposed for shear response analysis, in which the dynamic stiffness of the shear hinge is divided into two stages based on a threshold. Following the discrete time transfer matrix method for multibody system dynamics (MS-DT-TMM), the mechanical model and mathematical expression of each tunnel element is derived first and then assembled for the whole tunnel system. A solution procedure is proposed to solve the shear dynamic response of immersed tunnels using the proposed multibody system model. It is shown that the MS-DT-TMM has the same computational accuracy as the finite element method (FEM) and the modeling process is more efficient and flexible when compared to FEM. Although the MS-DT-TMM discussed herein is only applied to shear response analysis, it can easily be extended to analyze axial force and bending moment of immersed tunnels leading to a complete, rapid yet accurate enough seismic analysis of immersed tunnels suitable for engineering practices.
\end{abstract}

\section{Introduction}

Immersed tunnels are large and complex underground structures built in soft soil. An immersed tunnel consists of precast tunnel segments and joints connecting adjacent segments, as shown in Fig. 1. The segment elements are typically made of reinforced concrete with high stiffness [1]. The joints, comprised of Gina gasket and shear keys, have significantly lower stiffness compared to the segment elements. However shear key failure under large shear forces due to transverse seismic loadings is more crucial that will lead to structural failure of the whole tunnel system [2]. Therefore it is essential to analyze seismic response of immersed tunnels in transverse directions to ensure their safety against earthquake attack.

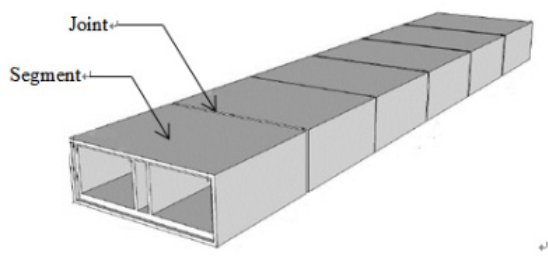

Fig 1. Schematic plot of an immersed tunnel

Many researchers have performed seismic analysis of immersed tunnels. For instance, Anastasopoulos et al. [3] FEM model investigated the seismic response of a $70 \mathrm{~m}-$ deep tunnel in a highly seismic region of Greece under 3D seismic excitation. In the FEM modeling of the immersed tunnel, the backbone curves of Gina gasket was bi-linear and the force vs. displacement relationship of shear keys in both transverses directions were considered to be the same. The FEM modeling procedure is complex and timeconsuming. Huang and Liu [4] proposed a modified displacement response method in the frequency domain considering the dependence of tunnel stiffness on the external loading frequency to achieve computational efficiency for seismic longitudinal response analysis of super-long immersed tunnels. Yuan et al. [5] employed a chain dynamic model of multibody system, i.e. a multirigid body, elastic damping hinge, and damping hinge model, is developed for dynamic analysis of immersed tunnel under longitudinal seismic excitations The study was only focused on longitudinal direction and the shear response under transverse loadings wasn't included for completion. However the seismic behavior of immersed tunnels along the longitudinal and transvers directions is very different, due to the different materials used in joints.

In this paper, MS-DT-TMM is further applied to shear seismic response analysis of immersed tunnels. A multirigid-body - shear hinge - viscous damping hinge model for shear seismic response analysis of immersed tunnels is developed. The mathematical model as well as transfer matrix of each element (i.e., rigid body element and shear joint element) and the whole system are derived. Finally, seismic shear responses of an example structure are computed using both the proposed MS-DT-TMM method and a FEM modeling procedure. Agreement of the responses obtained from these two methods is observed indicating the computational accuracy of MS-DT-TMM.

\footnotetext{
* Corresponding author:wpp8872@163.com
} 


\section{Multibody Dynamics Model for Shear Seismic Response of Immersed Tunnel}

Assumptions were made first to establish a simple yet reasonable mechanical model for shear seismic response analysis of immersed tunnels.

Based on the multi-rigid-body discretization idea, an immersed tunnel can be discretized into a series of rigid bodies with each element being modeled as a rigid body.

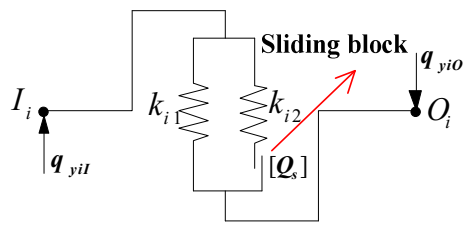

(a) Mechanical model
The joint is idealized as a shear hinge model and a bilinear elastic-plastic material model as shown in Fig. 2. The shear hinge model is comprised of two spring elements ( $k_{i 1}$ and $\left.k_{i 2}\right)$ and a slider. The slider connects with spring $\mathrm{k}_{\mathrm{i} 2}$ in series and then with $\mathrm{k}_{\mathrm{i} 1}$ in parallel. The shear stiffness of the joint at these two phases is idealized using a bi- linear model as illustrated in Fig. 2 (b), where $\mathrm{Q}_{\mathrm{s}}$ is the threshold of shear key.

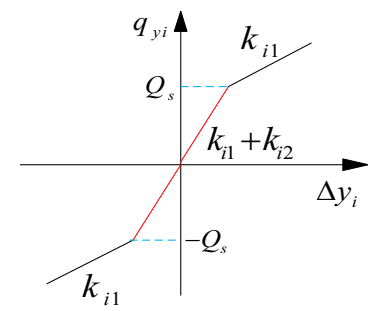

(b) Material model

Fig 2. Shear hinge model

Viscous damping hinge is employed to consider the soil-structure shear interaction. The viscous damping force between the segment element and soil is given by:

$$
f_{i \mathrm{y}}=-c_{i}^{\prime} \dot{y}_{i C}
$$

Where $f_{\text {iy }}$ is the viscous damping force between the $i^{\text {th }}$ segment element and soil, $c_{i}^{\prime}$ is the corresponding viscous damping coefficient; and $\dot{y}_{i C}$ is the shear velocity at the gravity centroid of the $i^{\text {th }}$ element.

For simplification purpose, a seismic input can be expressed in terms of a series of sine harmonic expressed as:

$$
F_{y}=P_{y} \sin (\omega t+\phi)
$$

where $F_{y}$ is shear earthquake loading, $P_{y}, \omega$ and $\phi$ are the loading's amplitude, frequency and initial phase, respectively.

Based on the assumptions above, a multi-rigid-body shear hinge - damping hinge model is established (see Fig. 3).

Following the principles of MS-DT-TMM when defining elements [6], $P_{i, j}$ is the connecting point between the $i^{\text {th }}$ and $j^{\text {th }}$ elements. Output point of the previous element is also the input point of the next element. The left end point and the right end point are two special points which are presented by $P_{\mathbf{1 , 0}}$ and $P_{n, n+1}$, respectively. oxy is the inertial Cartesian coordinate system and $o_{i} x_{i} y_{i}$ $(i=1,3, \ldots, n)$ are the element coordinate system of the $i^{\text {th }}$ rigid body. $C_{i}$ shows the gravity center of the $i^{\text {th }}$ rigid body element.

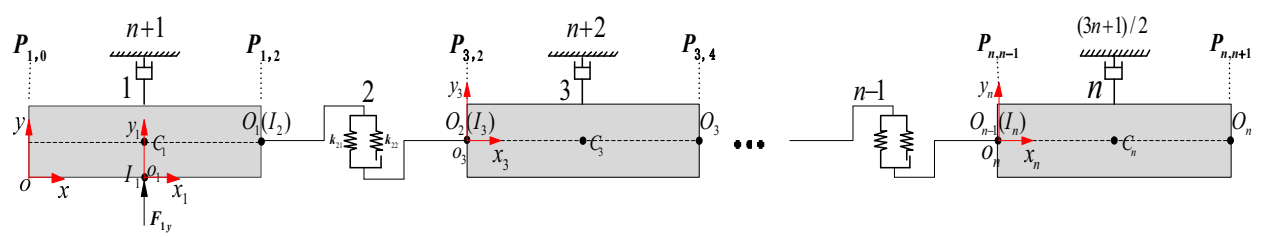

Fig 3. Multibody dynamics model of immersed tunnel

\section{Mathematical Model and Formulae}

\subsection{State Vectors}

A state vector of a connection point $P_{i, j}$ in a chain multibody system is defined by its position coordinate and force (see in Fig. 2) as:

$$
Z_{i, j}=\left[\begin{array}{lll}
y & q_{y} & 1
\end{array}\right]_{i, j}^{T}
$$

where the subscript $i$ is the serial numbers of the rigid body element and $j$ is the adjacent hinge element. $y$ is the position coordinate of the connection point along the $y$ axis with respect to the inertial reference; $q_{y}$ is the shear force of the point in the same reference system. "1" has no physical meaning but to match the transfer matrix form as discussed next. It also provides convenience of matrix operations.

\subsection{Transfer Matrices of Elements}

\section{Transfer Matrix of the first and the ith Rigid Body} Element

When establishing the transfer equation of the elements, transformation equations of the key points coordinates between these two systems needs to be established as listed in Table 1. 
Table1. Transformation equations of coordinates between the two coordinate systems

\begin{tabular}{|c|c|c|c|}
\hline Points & Initial coordinate & $\begin{array}{c}\text { Element } \\
\text { coordinate }\end{array}$ & Correlation equations \\
\hline Input point $I_{i}$ & $y_{1 I}$ & $y_{1 I}^{\prime}$ & $y_{1 I}=y_{1 I}^{\prime}$ \\
\hline Output point $O_{i}$ & $y_{1 O}$ & $y_{1 I O}^{\prime}$ & $y_{1 O}=y_{1 I}+y_{1 I O}^{\prime}$ \\
\hline Centroid $C_{i}$ & $y_{1 C}$ & $y_{1 I C}^{\prime}$ & $y_{1 C}=y_{1 I}+y_{1 I C}^{\prime}$ \\
\hline
\end{tabular}

To pass on the state vector from one element to another, both acceleration and velocity responses in transfer equations are expressed in terms of position coordinate $y$.

The shear acceleration $\ddot{y}_{I}$ and velocity $\dot{y}_{I}$ of the input point of a element at the time instant $t_{s}$ is represented as a linear function of its position coordinate ${ }^{y_{I}}$ :

$$
\begin{aligned}
& \ddot{y}\left(t_{s}\right)=A y\left(t_{s}\right)+B\left(t_{s}\right) \\
& \dot{y}\left(t_{s}\right)=C y\left(t_{s}\right)+D\left(t_{s}\right)
\end{aligned}
$$

where $A, B, C$ and $D$ are integration parameters at time $t_{s}$ determined based on the previous state $t_{s-1}$ and the integration method being adopted, which is the Newmark$\beta$ method in this study[7]:

$$
A=\frac{1}{\beta \Delta T^{2}}
$$

$$
\begin{gathered}
B\left(t_{s}\right)=-A\left[y\left(t_{s-1}\right)+\dot{y}\left(t_{s-1}\right) \Delta T+\left(\frac{1}{2}-\beta\right) \ddot{y}\left(t_{s-1}\right) \Delta T^{2}\right] \\
C=\frac{\gamma}{\beta \Delta T} \\
D\left(t_{s}\right)=\dot{y}\left(t_{s-1}\right)+(1-\gamma) \ddot{y}\left(t_{s-1}\right) \Delta T+\gamma B\left(t_{s}\right) \Delta T
\end{gathered}
$$

The integration constants $\gamma$ and $\beta$ are set to $1 / 2$ and $1 / 4$ respectively herein.

\begin{tabular}{|c|c|c|c|c|c|c|c|c|c|}
\hline $\begin{array}{l}\text { Dynamic } \\
\text { equilibrium } \\
\text { equation }\end{array}$ & \multicolumn{3}{|c|}{$\begin{array}{l}\text { The first element } \\
\qquad(i=1)\end{array}$} & \multicolumn{6}{|c|}{$\begin{array}{l}\text { The ith element } \\
\quad(i=3,5, \ldots, n)\end{array}$} \\
\hline About centroid & \multicolumn{3}{|c|}{$m_{1} \ddot{y}_{1 C}=q_{y 1 I}-q_{y 1 O}+F_{1 y}+f_{1 y}$} & \multicolumn{6}{|c|}{$m_{i} \ddot{y}_{i C}=q_{y i I}-q_{y i O}+f_{i y}$} \\
\hline $\begin{array}{l}\text { About input } \\
\text { point }\end{array}$ & \multicolumn{3}{|c|}{$\begin{array}{l}m_{1}\left(y_{1 I}^{\prime \prime}+y_{1 I C}^{\prime} "\right)= \\
q_{y 1 I}-q_{y 1 O}+F_{1 y}-c_{i}^{\prime}\left(y_{1 I}^{\prime}+y_{1 I C}^{\prime}{ }^{\prime}\right)\end{array}$} & \multicolumn{5}{|c|}{$\begin{array}{l}m_{i}\left(y_{i I}^{\prime \prime}+y_{i I C}^{\prime} "\right)= \\
q_{y i I}-q_{y i O}-c_{i}^{\prime}\left(y_{i I}^{\prime}+y_{i I C}^{\prime}\right)\end{array}$} & (16) \\
\hline $\begin{array}{c}\text { After } \\
\text { linearization }\end{array}$ & \multicolumn{3}{|c|}{$\begin{aligned} q_{y 1 O}= & \left(-m_{1} A-c_{1}^{\prime} C\right) y_{1 I}+q_{y 1 I} \\
& -m_{1} B_{1 I}-c_{1}^{\prime} D_{1 I}+F_{1 y}\end{aligned}$} & \multicolumn{6}{|c|}{$\begin{aligned} q_{y i O}= & \left(-m_{i} A-c_{i}^{\prime} C\right) y_{i I}+q_{y i I} \\
& -m_{i} B_{i I}-c_{i}^{\prime} D_{i I}\end{aligned}$} \\
\hline $\begin{array}{l}\text { Transfer } \\
\text { function }\end{array}$ & $\begin{array}{l}Z_{1,2}=U_{1} Z_{1,0} \\
=\left[\begin{array}{cc}1 & 0 \\
-m_{1} A-c_{1}^{\prime} C & 1 \\
0 & 0\end{array}\right.\end{array}$ & $\left.\begin{array}{c}y_{1 I O}^{\prime} \\
-m_{1} B_{1}-c_{1}^{\prime} D_{1}+F_{1 y} \\
1\end{array}\right]$ & $\begin{array}{l}Z_{1,0} \\
(19)\end{array}$ & $\begin{array}{l}Z_{i, i+1} \\
=[-l\end{array}$ & $\begin{array}{c}=U_{i} Z_{i, i-1} \\
1 \\
m_{i} A-c_{i}^{\prime} C \\
0\end{array}$ & $\begin{array}{l}0 \\
1 \\
0\end{array}$ & $-r$ & $\begin{array}{l}y_{i I O}^{\prime} \\
l_{i} B_{i}-c_{i}^{\prime} D_{i} \\
\quad 1\end{array}$ & $\int_{(20)}$ \\
\hline Transfer matrix & $U_{1}=\left[\begin{array}{c}1 \\
-m_{1} A-c_{1}^{\prime} C \\
0\end{array}\right.$ & $\begin{array}{cc}0 & y_{1 O O}^{\prime} \\
1 & -m_{1} B_{1}-c_{1}^{\prime} D_{1}+F_{1} \\
0 & 1\end{array}$ & $\left.F_{1 y}\right]$ & $U_{i}=$ & $=\left[\begin{array}{c}1 \\
-m_{i} A-c_{i}^{\prime} \\
0\end{array}\right.$ & & U & $\begin{array}{r}y_{i I O}^{\prime} \\
-m_{i} B_{i}-c \\
1\end{array}$ & $\left.{ }_{i} D_{i}\right]$ \\
\hline
\end{tabular}

The derivation of the transfer matrices of the first and the $i^{\text {th }}$ rigid body elements is shown in Table 2 which starts with the establishment of the dynamic equilibrium equations.

Table2. Derivation of transfer matrices of the $i^{\text {th }}$ rigid body elements

Transfer Matrix of the $\boldsymbol{i}^{\text {th }}$ Shear Hinge Element

The derivation of the transfer matrices of shear hinge elements corresponding to these two modeling phases are shown in Table 3 in tow columns, where $k_{i 1}$ and $k_{i 2}$ are the springs' stiffness; and $L_{i 0}$ is the initial springs' length. 
Table3. Derivation of transfer matrices of the ith $(i=2,4, \ldots, n-1)$ shear hinge element

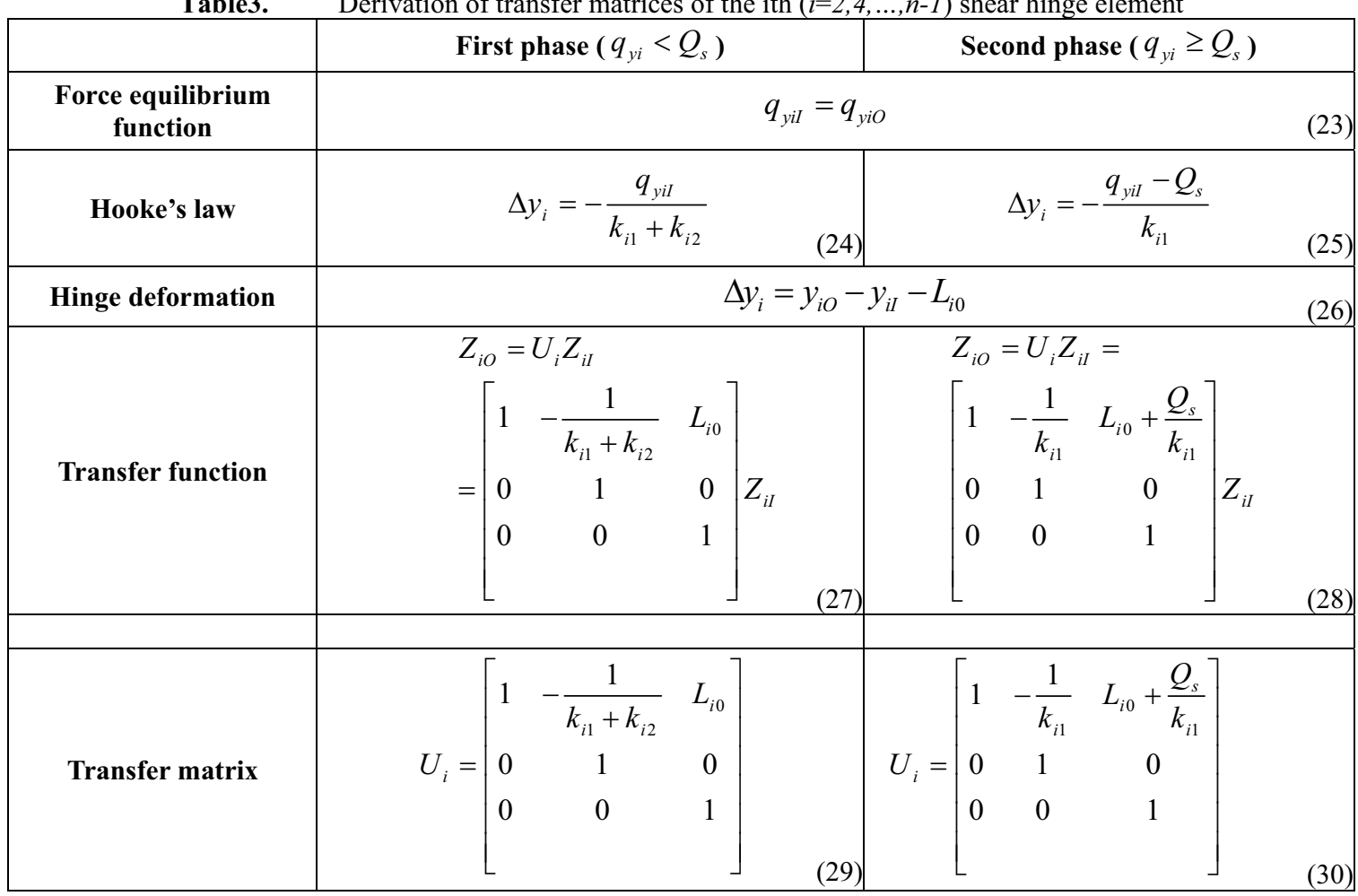

\subsection{Transfer Equation of the System}

An immersed tunnel is a system composed of a number of rigid-body elements connecting through joints modeled as hinge elements. The transfer equation of the each element is defined previously which can be written as a general form as:

$$
Z_{n, n+1}=U_{n} Z_{n, n-1}=U_{n} U_{n-1} Z_{n-2, n-1}=\ldots=U_{i} U_{i-1} \ldots U_{2} U_{1} Z_{1,0}=U Z_{1,0}
$$

where $Z_{n, n-1}$ and $Z_{n, n+1}$ are the state vectors of input and output points of the $n^{\text {th }}$ element. $U_{i}(i=1,2, \ldots, \mathrm{n})$ is the transfer matrices of the element shown Tables 2 and 3 in the last row for the rigid body elements and hinge elements respectively. The transfer matrix of entire tunnel system comprising of rigid body elements and hinge elements being serially connected can then be derived as

$$
U=U_{i} U_{i-1} \ldots U_{2} U_{1}
$$

Eqn. (32) reveals that the system transfer matrix of the chain multibody system for immersed tunnels can be assembled easily as the product of all the element transfer matrices of the system based on the multibody system dynamics.

\subsection{Dynamic Response Solution of the System}

The transfer matrix and the transfer function of the immersed tunnel system developed herein represent its shear response when subjected to a transverse load. To solve the tunnel response using the transfer equation, boundary and initial conditions need to be applied. In this case, free boundary conditions are applied at both end points of the tunnel model (i.e. non shear force at the input point of the first element and the output point of the last element, so $q_{y 1,0}=0$ and $q_{y n, n+l}=0$ ) and the state vectors of the these two points become:

$$
\begin{aligned}
Z_{1,0} & =\left[\begin{array}{lll}
y & 0 & 1
\end{array}\right]_{1,0}^{T} \\
Z_{n, n+1} & =\left[\begin{array}{lll}
y & 0 & 1
\end{array}\right]_{n, n+1}^{T}
\end{aligned}
$$

Then applying zero response initial conditions at time t0 into the system transfer Eqn. (31), we obtain the Eqn. (33) as shown:

$$
Z_{n, n+1}=\left[\begin{array}{lll}
y & 0 & 1
\end{array}\right]_{n, n+1}^{T}=U Z_{1,0}=U\left[\begin{array}{lll}
y & 0 & 1
\end{array}\right]_{1,0}^{T}
$$

From the process of solving mathematical model, the dynamic response of system at any time is gained by composing MATLAB program. What's more, if the number of elements increase, the transfer matrix of each element and system needn't to be deduced again. The system dynamic response at any time is efficiently gained by adding the corresponding transfer matrices in MATLAB program.

Comparing the seismic responses obtained from the proposed MS-DT-TMM and the FEM method, they are in a good agreement with the traditional FEM. So the dynamic and mathematical models of multibody system are suitable for aseismic design of immersed tunnel.

\section{Numerical Example}

To validate of the aforementioned MS-DT-TMM when being applied to solve shear dynamic response of immersed tunnels, a numerical example of an immersed tunnel model with two rigid body elements connecting by one joint is presented in this section (see Fig. 4). 


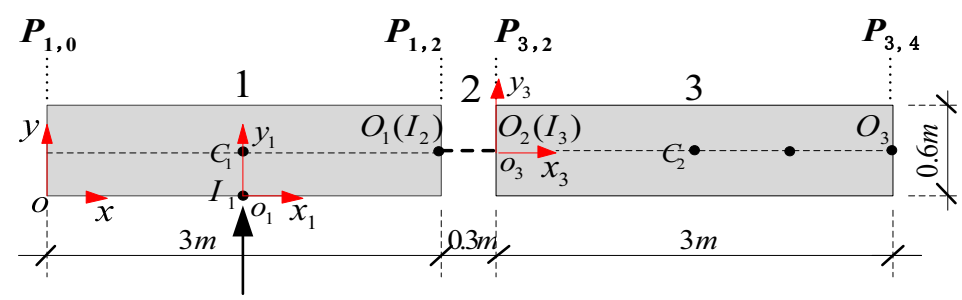

Fig 4. Mechanical model of the example

The input sine excitation is assumed as $F_{1 y}=500 \times 10^{3} \sin t$ with an input frequency of $\frac{1}{2 \pi}$.

The shear force threshold of the hinge is set as $Q_{s}=200 \times 10^{3} \mathrm{kN}$. The final structural parameters of each element are listed in Tables 4. The initial conditions at time $t_{0}=0$ are listed in Table 5 .
The responses solved by the MATLAB program based on the proposed MS-DT-TMM is then compared with those obtained using ABAQUS solution of the same example tunnel model. The mechanical models of the numerical example using MS-DT-TMM and ABAQUS are shown in Figs. 5 7, respectively.

Table4. Parameters of each element

\begin{tabular}{|c|c|c|c|c|}
\hline $\begin{array}{c}\text { Element } \\
\text { number }\end{array}$ & $\begin{array}{c}\text { Length } \\
/ \mathbf{m}\end{array}$ & $\begin{array}{c}\text { Width } \\
/ \mathbf{m}\end{array}$ & $\begin{array}{c}\text { Mass } \\
/ \mathbf{k g}\end{array}$ & $\begin{array}{c}\text { Damping hinge } \\
\left.\text { parameter } c_{i}^{\prime} \mathbf{( k g} / \mathbf{s}\right)\end{array}$ \\
\hline 1,3 & 3 & 0.6 & $1.366 e 5$ & $1.22 e 4$ \\
\hline $\begin{array}{c}\text { Element } \\
\text { number }\end{array}$ & $\begin{array}{c}\text { Initial length } \\
l_{0}(m)\end{array}$ & Stiffness $k_{21}(\mathrm{~N} / \mathrm{m})$ & Stiffness $k_{22}(\mathrm{~N} / \mathrm{m})$ \\
\hline 2 & 0.3 & \multicolumn{2}{|c|}{$1.74 \mathrm{e} 7$} & $1.74 \mathrm{e} 7$ \\
\hline
\end{tabular}

Table5. Initial conditions

\begin{tabular}{|c|c|c|c|c|}
\hline Element number & $\begin{array}{c}\text { Input point } \\
\text { position } \\
y_{i I}(\mathbf{m})\end{array}$ & $\begin{array}{c}\text { output point } \\
\text { position } \\
y_{i O} \mathbf{( m )}\end{array}$ & $\begin{array}{c}\text { Velocity } \dot{y}_{i I} \\
\mathbf{( m / s )}\end{array}$ & $\begin{array}{c}\text { Velocity } \dot{y}_{i O} \\
\mathbf{( m / s )}\end{array}$ \\
\hline 1 & 0 & 0.3 & 0 & 0 \\
\hline 2,3 & 0.3 & 0.3 & 0 & 0 \\
\hline
\end{tabular}

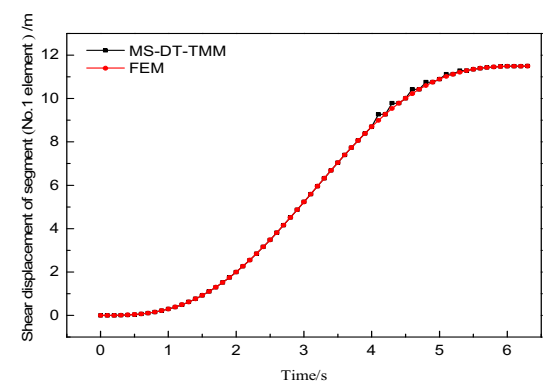

(a) No. 1 elemen

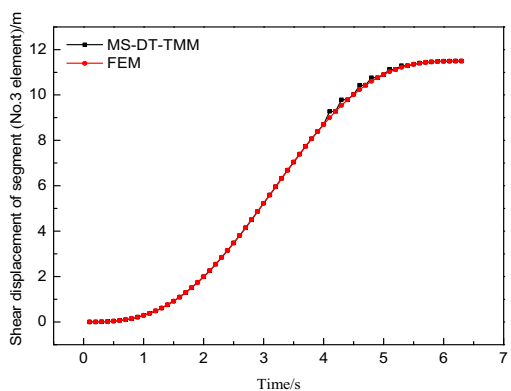

(b) No. 3 element

Fig 5. Time history of shear displacement of elements 


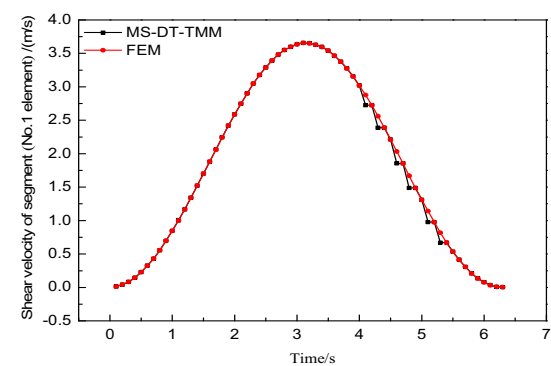

(a) No. 1 element

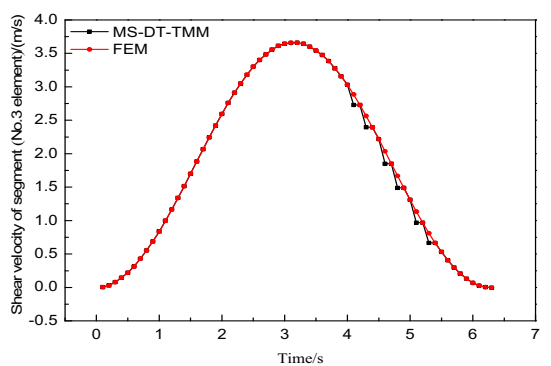

(b) No. 3 element

Fig 6. Time history of shear velocity of elements

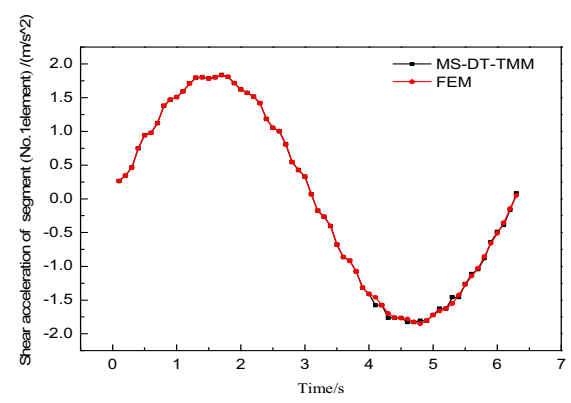

(a) No. 1 el

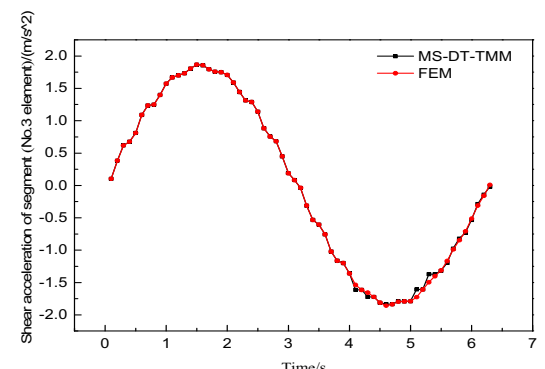

(b) No. 3 element

Fig 7. Time history of shear accelation of elements

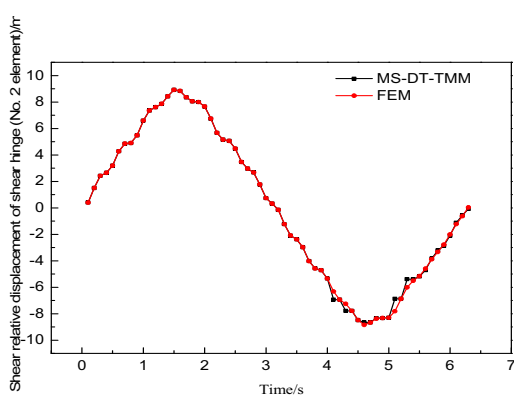

(a) shear relative displacement

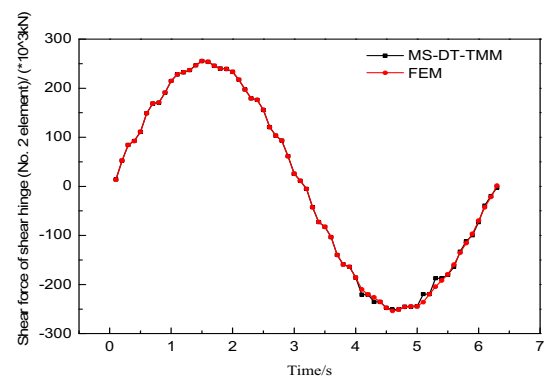

(b) shear force

Fig 8. Dynamic response of No. 2 element

\section{Conclusions}

Comparing the seismic responses obtained from the proposed MS-DT-TMM and the FEM method, it is concluded that: The solutions obtained from MS-DTTMM are in a good agreement with the traditional FEM. So the dynamic and mathematical models of multibody system are suitable for aseismic design of immersed tunnel. The multibody dynamic modeling process for immersed tunnel under earthquake is simple and flexible. MS-DTTMM has the advantages such as low orders, small memorizing size and high calculation efficiency and so on. The transfer matrices for typical rigid body element and shear hinge element deduced in this paper can be assembled into the whole transfer matrix for an immersed tunnel with arbitrary length. If MS-DT-TMM are expanded to shear seismic response analysis for long and ultra-long immersed tunnels, there will have broad application prospects.

\section{Acknowledgement}

The research has been supported by the First Class Professional Construction Pproject - Architectural Engineering Technology, Shanghai Municipal Education Commission (Z110-1003-20-001).

\section{References}

1. ITA Working Group. Immersed and Floating Tunnels (Report No. 007/OCT2011). International Tunneling and Underground Space Association (ITA) (2011).

2. I. Anastasopoulos, N. Gerolymos, V. Drosos, T. Georgarakos, R. Kourkoulis and G. Gazetas. Bull Earthquake Engineering, 6(2): 213-239 (2008)

3. I. Anastasopoulos, N. Gerolymos, V. Drosos, R. Kourkoulis and T. Georgarakos. Journal of Geotechnical and Geoenvironmental Engineering, 133(9):1067-1090 (2007) 
4. M. Huang and H. Liu. Chinese Journal of Geotechnical Engineering,37(11):1971-1978 (2015)

5. Y. Yuan, Z. Shen, and H. Yu. Engineering Mechanics, 32: 76-83 (2015).

6. X. Rui, B. He, Y. Lu, W. Lu and G. Wang. Multibody System Dynamics, 14(4): 317-344 (2005)

7. A. Kumar and T. Sankar. Computer \& Structures, 23(4): 545-552 (1986) 